\title{
Real-World Efficacy and Safety of Lenvatinib in Korean Patients with Advanced Hepatocellular Carcinoma: A Multicenter Retrospective Analysis
}

\author{
Jaekyung Cheon $^{\mathrm{a}}$ Hong Jae Chon ${ }^{\mathrm{b}}$ Yeonghak Bang ${ }^{\mathrm{c}}$ Neung Hwa Park ${ }^{\mathrm{a}}$ \\ Jung Woo Shin ${ }^{a}$ Kang Mo Kim ${ }^{d}$ Han Chu Lee ${ }^{d}$ Jooho Lee \\ Changhoon $\mathrm{Yoo}^{\mathrm{C}}$ Baek-Yeol Ryoo ${ }^{\mathrm{C}}$ \\ a Department of Internal Medicine, Ulsan University Hospital, University of Ulsan College \\ of Medicine, Ulsan, South Korea; ${ }^{b}$ Departments of Medical Oncology, CHA Bundang \\ Medical Center, CHA University School of Medicine, Seongnam, South Korea; ${ }^{\mathrm{C}}$ Department \\ of Oncology, Asan Medical Center, University of Ulsan College of Medicine, Seoul, South \\ Korea; ${ }^{\mathrm{d} D e p a r t m e n t}$ of Gastroenterology, Asan Medical Center, University of Ulsan College \\ of Medicine, Seoul, South Korea; ${ }^{e}$ Departments of Gastroenterology and Hepatology, CHA \\ Bundang Medical Center, CHA University School of Medicine, Seongnam, South Korea
}

\section{Keywords}

Lenvatinib $\cdot$ Hepatocellular carcinoma $\cdot$ Liver cancer $\cdot$ Real-world conditions

\begin{abstract}
Introduction/Objective: Lenvatinib demonstrated efficacy and safety in patients with advanced hepatocellular carcinoma (HCC) in the randomized phase III REFLECT trial. Considering the discrepancies in patients between clinical trial data and daily practice, an account of practical experience is needed. Methods: We conducted a multicenter retrospective analysis in which 3 tertiary referral centers participated. A total of 92 patients with advanced HCC treated with lenvatinib between September 2018 and January 2020 were analyzed. Results: Lenvatinib was used as the first-line therapy for 67 (72.8\%) patients, and for 25 (27.2\%) patients previously treated with other systemic therapy including immune checkpoint inhibitors. At the time of initiation of lenvatinib, 74 (80.4\%) and 18 (19.6\%) patients were classified as Child-Pugh
\end{abstract}

Jaekyung Cheon and Hong Jae Chon should be considered co-first authors. Changhoon Yoo and BaekYeol Ryoo should be considered co-corresponding authors. 
$A$ and $B$, respectively. Thirty-five patients (38.0\%) had extensive disease that would have excluded them from the REFLECT trial. In the Child-Pugh A group, the response rate graded according to the Response Evaluation Criteria in Solid Tumors v1.1 was $21.1 \%$, median progression-free survival (PFS) was 4.6 (95\% confidence interval $[\mathrm{Cl}] 3.1-6.1)$ months, and overall survival (OS) was 10.7 (95\% Cl 4.8-16.5) months for patients treated with first-line lenvatinib $(n=57)$. With second- or later-line lenvatinib $(n=17)$, median PFS and OS were $4.1(95 \% \mathrm{Cl}$ 3.1-5.1) and $6.4(95 \% \mathrm{Cl} 5.1-7.7)$ months, respectively. In the Child-Pugh B group ( $n=18)$, median PFS and OS were $2.6(95 \% \mathrm{Cl} 0.6-4.6)$ and $5.3(95 \% \mathrm{Cl} 2.0-8.5)$ months, respectively. The most common grade 3-4 toxicities were hyperbilirubinemia $(n=8 ; 8.7 \%)$, AST elevation $(n=$ $6 ; 6.5 \%)$, and diarrhea $(n=5 ; 5.4 \%)$ across all study patients. Conclusions: In this real-world study, lenvatinib was found to be well tolerated and effective in more heterogeneous HCC patient populations.

\section{Introduction}

Hepatocellular carcinoma (HCC) has been reported to be one of the most frequent causes of cancer-related deaths globally and in Korea [1,2]. HCC is an extremely heterogeneous disease and the selection of treatment strategy may vary depending on the tumor burden, the degree of underlying liver cirrhosis, and the patient's performance status. For patients with early-stage HCC, curative approaches including surgical resection, liver transplantation, and ablative techniques offer chances of long-term survival. However, systemic therapy is the mainstay of treatment in patients with advanced- and intermediate-stage disease unsuitable for, or refractory to, transarterial chemoembolization (TACE).

Sorafenib was the first drug to show improved survival in patients with advanced HCC, and it was established as a standard first-line systemic treatment based on the success of pivotal phase III trials [3, 4]. However, because global phase III trials of sunitinib, brivanib, and linifanib failed to demonstrate a survival benefit compared to sorafenib, there has been very limited progress in the treatment of advanced HCC over the past 10 years [5-7].

Lenvatinib is an oral multikinase inhibitor that targets vascular endothelial growth factor receptors (VEGFRs) 1-3, fibroblast growth factor receptors (FGFRs) 1-4, platelet-derived growth factor receptor $\alpha$ (PDGFR- $\alpha$ ), c-Kit, and RET [8, 9]. Based on the promising efficacy report from the phase 2 study [10], lenvatinib was subsequently investigated as a first-line therapy against advanced HCC in the randomized phase III REFLECT trial, as a head-to-head comparison with sorafenib. In the REFLECT trial, lenvatinib showed noninferiority to sorafenib in overall survival (OS), with a clinically meaningful improvement in progressionfree survival (PFS), and objective response rate (ORR) [11]. Current international guidelines recommend lenvatinib as the first-line therapy for patients with advanced HCC [12-15]. Realworld evidence is important in HCC because of the discrepancies in patient characteristics between clinical trials and daily practice. It is important to note that patients with a huge tumor burden (i.e., the tumor occupies $\geq 50 \%$ of the liver), bile duct invasion, main portal vein invasion, and poor liver function were not included in the REFLECT trial, even though such patients exhibit a larger unmet need.

Therefore, we performed a multicenter retrospective analysis to evaluate the efficacy and safety of lenvatinib for patients with advanced HCC in the real-world setting. 


\section{Materials and Methods}

Patients

This investigation was a retrospective, multicenter, noncomparative, observational study of patients treated with lenvatinib for advanced HCC. Eligible patients presented with HCC, confirmed by a pathologic or noninvasive assessment according to criteria of the American Association for the Study of Liver Diseases, Barcelona Clinic Liver Cancer (BCLC) stages B or C categorizations [16], and Child-Pugh class A or B. Patients who were followed up at the clinic at least once after the administration of lenvatinib were included in this analysis.

Data on age, sex, etiology, Eastern Cooperative Oncology Group (ECOG) performance status, $\alpha$-fetoprotein (AFP) levels, maximum diameter of intrahepatic lesions, Child-Pugh score, and BCLC stage at the time of starting lenvatinib were collected by reviewing medical records and images.

\section{Treatment and Assessment}

The standard dosing schedule of lenvatinib used in the REFLECT trial $(8 \mathrm{mg} /$ day to patients weighing $<60 \mathrm{~kg}$ and $12 \mathrm{mg} /$ day to those $\geq 60 \mathrm{~kg}$ ) was recommended. However, modification of the starting dose was allowed depending on the clinical situation at the discretion of the attending physicians. Dose interruptions or reductions were made according to the protocol of the REFLECT trial. Tumor assessment using CT or MRI was performed every 6-8 weeks, or whenever there was a sign or symptom suggesting tumor progression. Tumor response was determined according to the Response Evaluation Criteria in Solid Tumors (RECIST) v1.1 [17]. Grades of adverse events (AEs) complied with the Common Terminology Criteria for Adverse Events (CTCAE) v4.03.

Statistical Analysis

PFS was defined as the length of time from the start of lenvatinib to the date of disease progression by RECIST v1.1, or death from any cause, whichever occurred first. OS was defined as the time from the initiation of lenvatinib treatment to death from any cause. Survival curves were estimated using the Kaplan-Meier method and compared using the log-rank test. $p<0.05$ was considered significant. SPSS v21.0 (IBM, Armonk, NY, USA) was used for all statistical analyses.

\section{Results}

\section{Patient Characteristics}

Baseline patient characteristics are summarized in Table 1. From September 2018 to January 2020, 92 patients altogether who met the inclusion criteria were included in this analysis, out of the 99 total patients who received lenvatinib. The median age was 60 years (range 19-81 years), and 71 of the patients (77.2 \%) were male. Hepatitis B virus was the most common etiology of HCC $(n=67,72.8 \%)$. The majority of patients were classified as Child-Pugh A $(n=74,80.4 \%)$ or BCLC stage C $(n=81,88.0 \%)$, while 18 patients $(19.6 \%)$ and 11 patients $(12.0 \%)$ had Child-Pugh B or BCLC stage B, respectively, at the time of initiation of lenvatinib. Sixty-one patients $(66.3 \%)$ had extrahepatic metastasis, with the most common metastatic site being the lungs $(n=46,50.0 \%)$, followed by the lymph nodes $(n=29,31.5 \%)$, and peritoneum $(n=12,13.0 \%)$. Macrovascular invasion was noted in 37 patients $(40.2 \%)$ and baseline serum AFP exceeded $200 \mathrm{ng} / \mathrm{mL}$ in 49 patients (53.3\%). This study included 35 (38.0\%) patients who did not meet the REFLECT inclusion criteria in terms of disease extent (bile duct invasion, $n=10$; main portal vein invasion, $n=15$; and tumor occupying $\geq 50 \%$ of the liver, $n=22$ ).

Lenvatinib was administered as first-line therapy to 67 (72.8\%) patients, second-line therapy to 14 (15.2\%), and third or fourth-line therapy to 11 (11.9\%) patients. Of the 25 patients who had received prior systemic therapy, sorafenib and regorafenib were the drugs administered to 18 (19.6\%) and 10 (10.9\%) patients, respectively, and immune checkpoint inhibitors (ICIs) to 15 (16.3\%, i.e., nivolumab to 8 , atezolizumab + bevacizumab to 6, and 
Table 1. Baseline characteristics of 92 patients

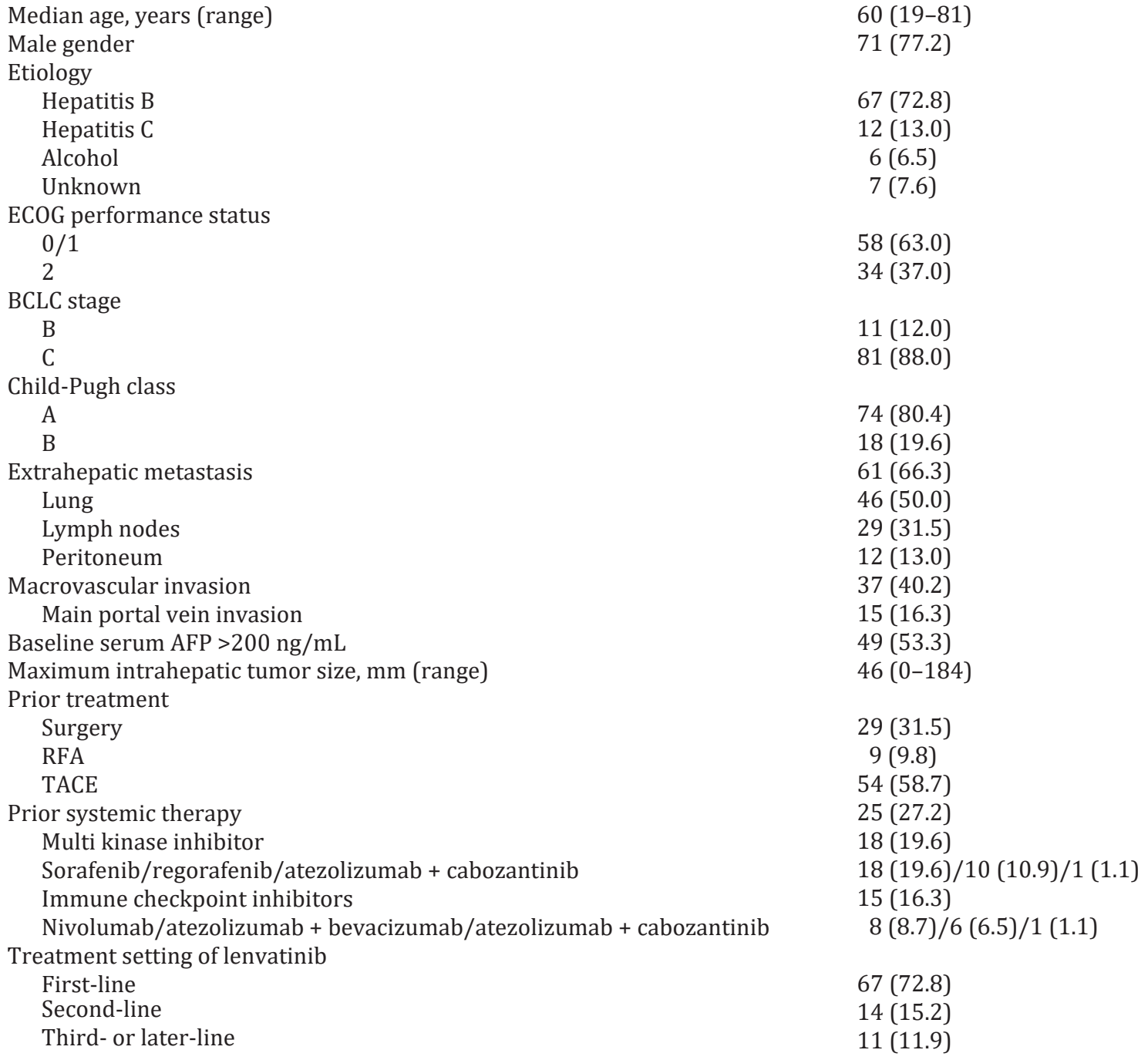

Values are expressed as $n(\%)$, unless otherwise indicated. ECOG, Eastern Cooperative Oncology Group; BCLC, Barcelona Clinic Liver Cancer; RFA, radiofrequency ablation; AFP, $\alpha$-fetoprotein; TACE, transcatheter arterial chemoembolization.

atezolizumab + cabozantinib to 1). There was no significant difference in key baseline characteristics between patients who received lenvatinib as first-line and second- or later-line treatment (online suppl. Table 1; for all online suppl. material, see www.karger.com/ doi/10.1159/000508901).

Starting doses of lenvatinib were: $12 \mathrm{mg}(n=32 ; 34.8 \%), 8 \mathrm{mg}(n=49 ; 53.3 \%)$, or $4 \mathrm{mg}$ daily $(n=11 ; 12.0 \%)$ daily. The starting dose of lenvatinib was reduced in 22 patients $(23.9 \%)$ because of a poor performance status $(n=14)$, old age $(n=6)$, or poor liver function $(n=2$, both with a Child-Pugh score of 9).

\section{Efficacy}

Of all the study patients, 13 achieved a partial response (PR), graded by RECIST v1.1, but none achieved a complete response, resulting in an ORR of $14.1 \%$. Stable disease (SD) and 


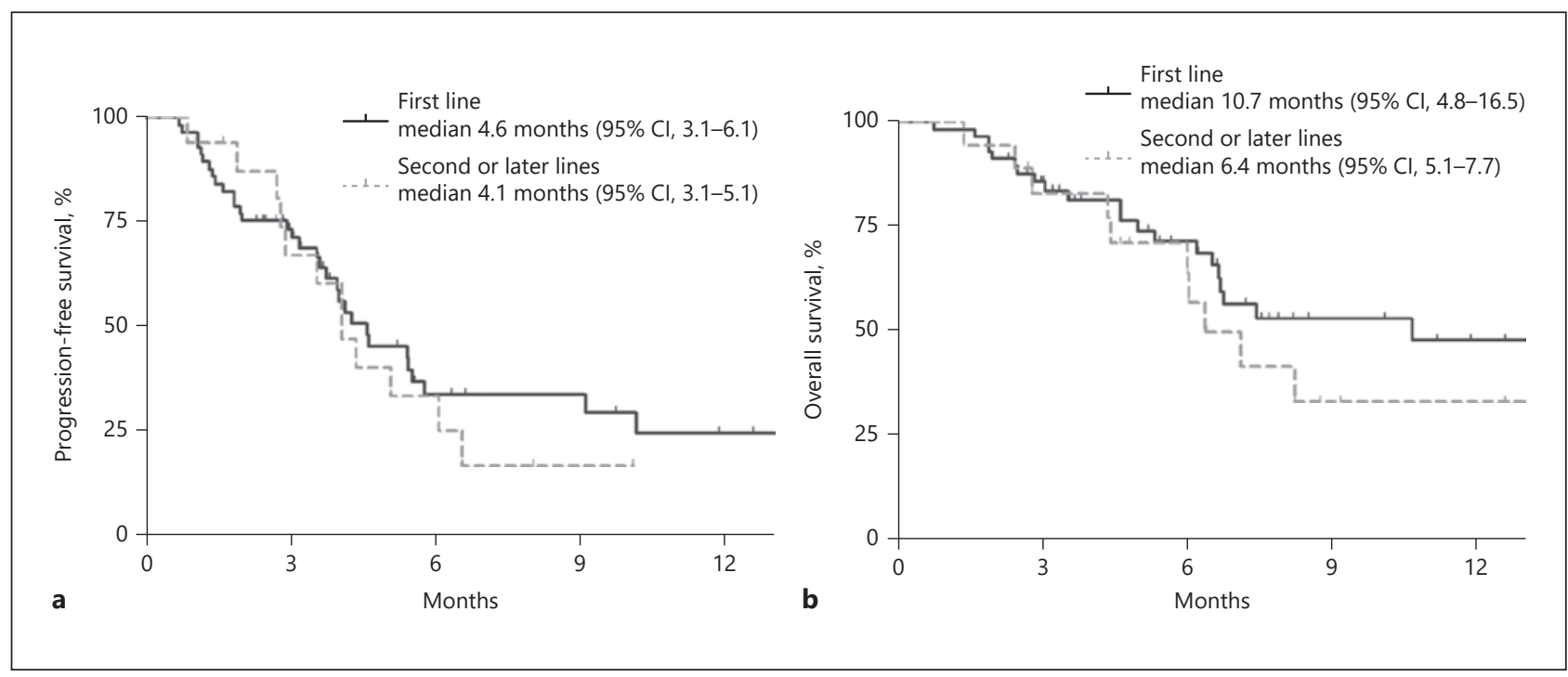

Fig. 1. Survival outcomes with lenvatinib in Child-Pugh A group, according to the line of therapy (first line vs. second or later lines). a Progression-free survival. b Overall survival.

progressive disease (PD) were the best responses, observed in $60(65.2 \%)$ and 19 (20.7\%) patients, respectively, and the disease control rate (DCR) was 79.3\%. The median follow-up duration was 6.6 months (95\% confidence interval [CI] 4.9-8.4 months), median PFS was 4.3 months (95\% CI 3.2-5.3 months), and median OS was 7.1 months (95\% CI 5.5-8.7 months). In patients who received lenvatinib as second- or later-line therapy $(n=25)$, the median OS from the start of first-line systemic therapy was 15.5 months (95\% CI 8.3-22.6 months).

Because of the heterogeneous nature of the population included in this study, efficacy outcomes were analyzed after stratification according to the treatment line of lenvatinib, the Child-Pugh class, and the BCLC stage when lenvatinib was initiated (Table 2).

In the Child-Pugh A group, first-line lenvatinib $(n=57)$ showed a median PFS of 4.6 months (95\% CI 3.1-6.1 months) and a median OS of 10.7 months (95\% CI 4.8-16.5 months). In patients receiving lenvatinib as a second- or later-line treatment $(n=17)$, median PFS and OS were 4.1 months (95\% CI 3.1-5.1 months) and 6.4 months (95\% CI 5.1-7.7 months), respectively. Despite the median PFS and OS being numerically better in patients with firstline lenvatinib compared to those with second- or later-line lenvatinib, the differences were not statistically significant ( $p=0.427$ for PFS and $p=0.728$ for OS; Fig. 1 ). However, the ORR was significantly higher in patients with first-line lenvatinib than in those with second- or later-line lenvatinib ( 21.1 vs. $0 \% ; p=0.039$ ). In the subgroups of patients who received prior ICIs $(n=11)$, the median PFS and OS with lenvatinib were 4.4 months (95\% CI 3.0-5.8 months) and 7.1 months (95\% CI 5.6-8.6 months), respectively. In the patients with an extensive tumor burden in the liver (main portal vein invasion, bile duct invasion, or a huge tumor involving $\geq 50 \%$ of the liver) that meant they were excluded from the REFLECT trial ( $n=28)$, the median PFS and OS were 4.0 months (95\% CI 3.5-4.5 months) and 6.0 months (95\% CI 4.5-7.5 months), respectively. The median OS was 13.7 months (95\% CI 4.7-22.6) in patients who were eligible according to the REFLECT trial criteria except for a history of systemic treatment $(n=46)$.

In the Child-Pugh B group ( $n=18)$, median PFS and OS were 2.6 months (95\% CI 0.6-4.6 months) and 5.3 months (95\% CI 2.0-8.5 months), respectively, and ORR was 5.6\%. When compared with the Child-Pugh A group, the patients in the Child-Pugh B group had a signifi- 
Fig. 2. Survival outcomes with lenvatinib in Child-Pugh B group.

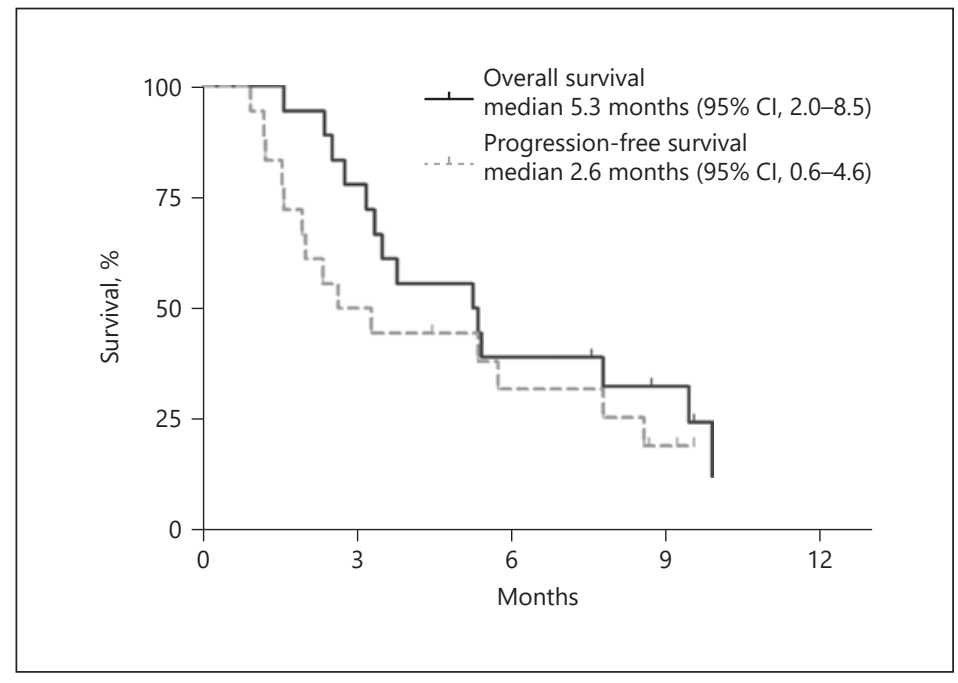

cantly poorer OS $(p=0.039)$ and numerically lower PFS $(p=0.377)$ and ORR $(p=0.451)$ (Fig. 2).

When patients were stratified according to BCLC stage, median PFS and OS with lenvatinib were 4.3 months (95\% CI 3.6-4.9 months) and 6.8 months (95\% CI 4.7-8.8 months), respectively, in patients with BCLC stage C $(n=81)$, and 7.8 and 7.8 months $(95 \%$ CIs not available), respectively, in patients with BCLC stage B $(n=11)$. ORR was 36.4 and $11.1 \%$ in BCLC stage B and C patients, respectively. Median PFS and OS were 4.1 months (95\% CI not available) and 6.7 months (95\% CI 1.5-11.9 months), respectively, in patients with ChildPugh A/BCLC stage B, and 4.4 months (95\% CI 3.7-5.0 months) and 7.4 months (95\% CI 3.3-11.5 months), respectively, in patients with Child-Pugh A/BCLC stage C (Fig. 3a, b). In patients with Child-Pugh B/BCLC stage B, median PFS and OS were 7.7 months (95\% CI not available) and 7.7 months (95\% CI not available), respectively, and 2.6 months (95\% CI 0.9-4.3 months) and 5.3 months (95\% CI 2.8-7.8 months), respectively, in those with ChildPugh B/BCLC stage C (Fig. 3c, d).

\section{Subsequent Therapy}

After disease progression on lenvatinib, 32 patients received subsequent treatment: sorafenib $(n=16)$, nivolumab $(n=14)$, cabozantinib $(n=1)$, or an investigational immunotherapeutic agent $(n=1)$. Patients who received postprogression treatment after lenvatinib had better baseline liver function (Child-Pugh A; 87.5 vs. 63.0\%; $p=0.035$ ) and less tumor burden in terms of intrahepatic tumor diameter ( $44 \mathrm{vs} .74 \mathrm{~mm} ; p=0.018$ ) at the time of start of lenvatinib (online suppl. Table 2).

\section{Safety Profile}

The profiles of AEs are listed in Table 3. The most common AEs of any grade were aspartate aminotransferase (AST) elevation ( $n=48 ; 52.2 \%)$, fatigue $(n=36 ; 39.1 \%)$, hyperbilirubinemia ( $n=24 ; 26.1 \%)$, and thrombocytopenia $(n=24 ; 26.1 \%)$. Twenty-seven $(29.3 \%)$ patients had grade 3-4 AEs and there were no treatment-related deaths. The most frequent grade 3-4 AEs were hyperbilirubinemia $(n=8 ; 8.7 \%)$, AST elevation $(n=6 ; 6.5 \%)$, diarrhea $(n=5 ; 5.4 \%)$, fatigue $(n=4 ; 4.3 \%)$, and hypertension $(n=4 ; 4.3 \%)$. In the Child-Pugh B group, grade $3-4$ AEs were reported in 5 patients $(27.8 \%)$, and hyperbilirubinemia $(n=4 ; 22.2 \%)$ was the most common. 


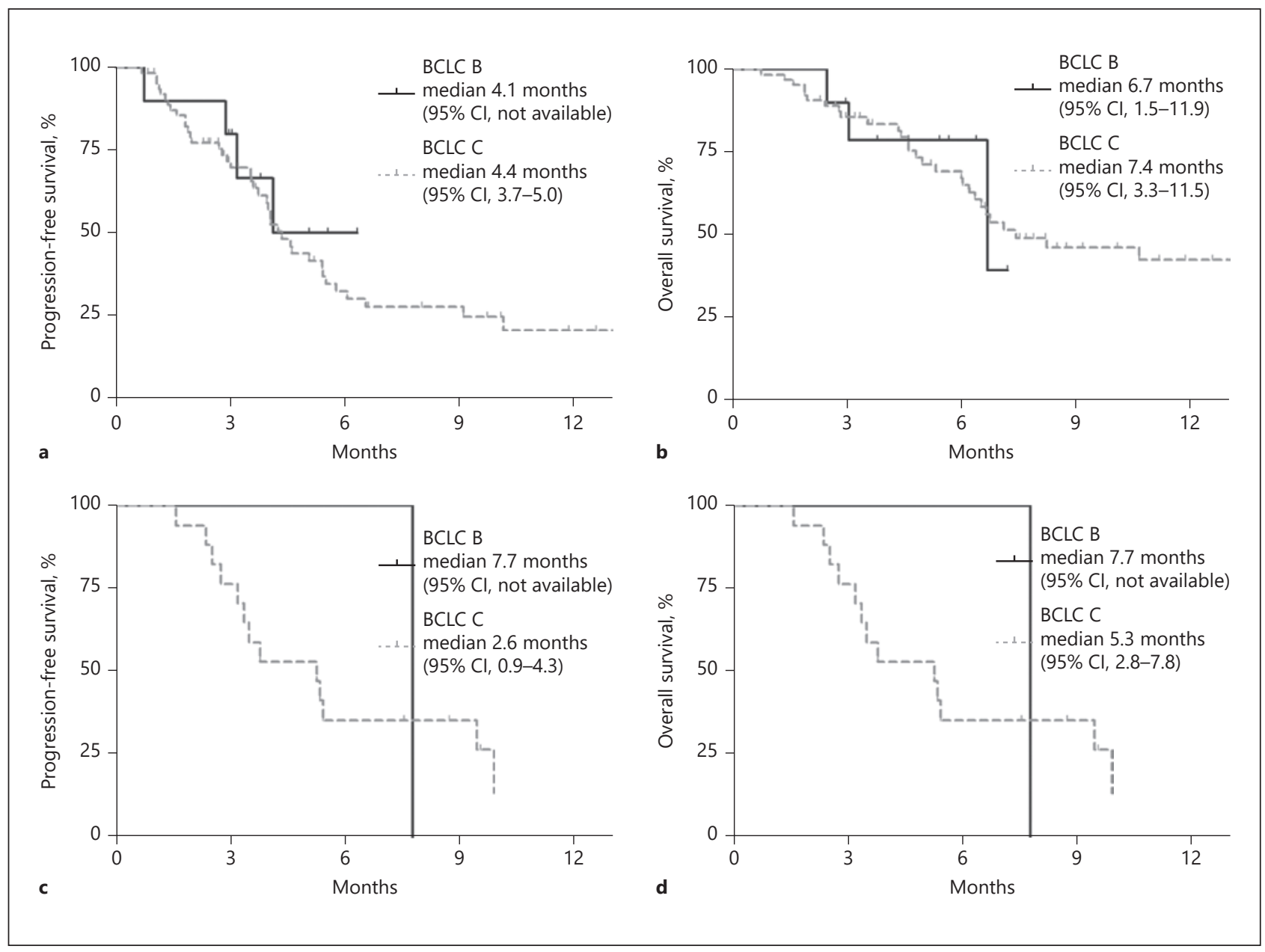

Fig. 3. Survival outcomes with lenvatinib according to BCLC stage. PFS (a) and OS (b) in Child-Pugh A patients. PFS (c) and OS (d) in Child-Pugh B patients. BCLC, Barcelona Clinic Liver Cancer.

At the time of analysis, lenvatinib was discontinued in 65 patients $(56.5 \%)$, and the most common cause for this was disease progression $(n=52 ; 80 \%)$. AEs were the reasons for the discontinuation of lenvatinib in 8 patients (12.3\%): a deterioration in liver function $(n=4 ; 2$ patients were Child-Pugh class B7 at the start of lenvatinib), generalized edema $(n=1)$, decreased renal function $(n=1)$, diarrhea $(n=1)$, and stomatitis $(n=1)$. The median duration of treatment was 2.8 months $(95 \% \mathrm{CI}, 0.0-5.9)$ for patients who stopped lenvatinib due to AEs.

\section{Discussion}

In this multicenter, retrospective analysis, the real-world efficacy and toxicities of lenvatinib were evaluated in 92 Korean patients with advanced HCC. The Child-Pugh A patients receiving first-line lenvatinib showed an ORR according to the RECIST v1.1 of $21.1 \%$, a median PFS of 4.6 months, and a median OS of 10.7 months. These outcomes seem to be numerically poorer than the results shown in the phase III REFLECT trial, which showed a median OS of 13.6 months and a median PFS of 7.3 months graded by masked independent imaging review 
Table 3. Safety profiles and treatment discontinuations

\begin{tabular}{|c|c|c|c|c|c|c|}
\hline & \multicolumn{2}{|l|}{ All $(n=92)$} & \multicolumn{2}{|c|}{ Child-Pugh A $(n=74)$} & \multicolumn{2}{|c|}{ Child-Pugh B $(n=18)$} \\
\hline & any grade & grade $3-4$ & any grade & grade $3-4$ & any grade & grade $3-4$ \\
\hline Any adverse event & $80(87.0)$ & 27 (29.3) & $63(85.1)$ & $22(29.7)$ & $15(83.3)$ & $5(27.8)$ \\
\hline Fatigue & $36(39.1)$ & $4(4.3)$ & $33(35.9)$ & $4(5.4)$ & $3(16.7)$ & 0 \\
\hline Anorexia & $21(22.8)$ & 0 & 19 (25.9) & 0 & $2(11.2)$ & 0 \\
\hline Nausea & $12(13.0)$ & $1(1.1)$ & $10(13.5)$ & 0 & $2(11.2)$ & $1(5.6)$ \\
\hline Vomiting & $4(4.3)$ & 0 & $4(5.4)$ & 0 & 0 & 0 \\
\hline Diarrhea & $10(10.9)$ & $5(5.4)$ & $8(10.8)$ & $4(5.4)$ & $1(5.6)$ & $1(5.6)$ \\
\hline Hypertension & $15(16.3)$ & $4(4.3)$ & $14(18.9)$ & $4(5.4)$ & $1(5.6)$ & 0 \\
\hline Mucositis & $2(2.2)$ & $1(1.1)$ & $2(2.8)$ & $1(1.4)$ & 0 & 0 \\
\hline Hand-foot syndrome & $4(4.4)$ & 0 & $2(2.7)$ & 0 & $2(11.2)$ & 0 \\
\hline Hypothyroidism & $6(6.5)$ & 0 & $5(6.8)$ & 0 & $1(5.6)$ & 0 \\
\hline Neutropenia & $6(6.5)$ & $1(1.1)$ & $5(6.9)$ & $1(1.4)$ & $1(5.6)$ & 0 \\
\hline Anemia & $17(18.4)$ & 0 & $10(13.5)$ & 0 & 7 (38.9) & 0 \\
\hline Thrombocytopenia & $24(26.1)$ & $2(2.2)$ & $19(25.7)$ & $2(2.7)$ & $5(27.8)$ & 0 \\
\hline AST elevation & $48(52.2)$ & $6(6.5)$ & $33(44.6)$ & $5(6.8)$ & $6(33.3)$ & $1(5.6)$ \\
\hline ALT elevation & $23(25.0)$ & 0 & $13(17.6)$ & 0 & $10(53.5)$ & 0 \\
\hline Hyperbilirubinemia & $24(26.1)$ & $8(8.7)$ & $16(21.6)$ & $4(5.4)$ & $8(44.4)$ & $4(22.2)$ \\
\hline Proteinuria & $14(15.2)$ & $1(1.1)$ & $14(18.9)$ & $1(1.4)$ & 0 & 0 \\
\hline \multicolumn{7}{|l|}{ Interruption and/ } \\
\hline or dose reduction & $22(23.9)$ & & $20(27.0)$ & & $2(11.1)$ & \\
\hline
\end{tabular}

Values express $n(\%)$. AST, aspartate aminotransferase; ALT, alanine aminotransferase.

according to RECIST v1.1 [11]. However, 37.8\% of our Child-Pugh A patient population had extensive tumor burdens including macrovascular invasion, bile duct invasion, or $\geq 50 \%$ liver involvement, and were therefore not eligible for inclusion to the REFLECT trial. Fifty-three percent of all study patients had AFP levels $>200 \mathrm{ng} / \mathrm{mL}$, compared to $46 \%$ in the REFLECT trial [18]. As these factors may portend a poorer prognosis in HCC patients, our efficacy results are likely in line with those of the REFLECT trial. In patients who met the REFLECT trial criteria except for a past history of systemic therapy, the median OS was 13.7 months, which is comparable with the outcomes of the REFLECT trial and previous retrospective study $[11,19]$. During our study period, lenvatinib was approved but not reimbursed by national health insurance in Korea, which placed a greater financial burden on patients compared to sorafenib. This resulted in physicians preferentially using lenvatinib for patients with a poorer anticipated prognosis, and thus a shorter treatment course. Taken together with our findings and the results of previous Japanese retrospective analyses which showed median PFS by modified RECIST of 5.1-7.4 months and ORR by modified RECIST of 33-61\% $[20,19,21-25]$, lenvatinib is considered effective for the management of advanced HCC. This is reinforced when our results are compared with those of the Korean subset of the GIDEON study for sorafenib [26], although cross-trial comparisons should be interpreted with caution. In that particular study, median OS and time to progression (TTP) with sorafenib were 8.5 and 2.5 months, respectively, with an ORR of $2.9 \%$, i.e., numerically worse than the current findings with lenvatinib.

Because lenvatinib was investigated as a first-line therapy in previous studies, there are no data based on the prospective trials for its use as salvage therapy after disease progression on prior systemic therapy. Interestingly, lenvatinib showed a similar PFS when used as a firstline or second-/later-line therapy (PFS, median 4.6 vs. 4.1 months), and a numerically better OS for patients treated with first-line lenvatinib (median 10.7 vs. 6.4 months) in our study. 
Although the number of patients was small $(n=15)$, our study population also included patients who had progressed on prior ICIs. As a similar PFS, regardless of therapeutic line of lenvatinib, was also shown in a previous study [21], this may indicate that sequential systemic therapy including lenvatinib may prolong the OS of patients with advanced HCC [27]. As atezolizumab plus bevacizumab has demonstrated superiority (in terms of PFS and OS) to sorafenib as a first-line therapy [28] and there is a strong possibility of positioning this combination as a new first-line therapy, our findings may indicate that lenvatinib has the potential to serve as a second-line therapy behind this novel regimen, although further studies in a large patient population are needed.

In our study, the Child-Pugh B patients showed poorer survival outcomes (PFS 2.6 months and OS 5.3 months) than the Child-Pugh A patients (PFS 4.4 months and OS 7.4 months). Our results with regard to the Child-Pugh B group are in line with previous studies on lenvatinib or sorafenib. In a previous Japanese retrospective study, the median OS of the Child-Pugh B group was 5.5 months [19]. In the GIDEON study, the median OS of Child-Pugh B patients receiving sorafenib was 5.2 months, which was worse than the 13.6 months for Child-Pugh A patients [29]. These findings support the notion that baseline liver function is a critical predictor of survival outcomes in patients with advanced HCC. Because the efficacy of lenvatinib in Child-Pugh B patients seems to be modest and hyperbilirubinemia was found to be more common in this group (any grade and grade 3-4: Child-Pugh A, 21.6 and 5.4\%; Child-Pugh B, 44.4 and $22.2 \%$, respectively), lenvatinib administration to Child-Pugh B patients should be judicious and employed only after assessing for the potential clinical benefit.

In the subgroup analysis of the Japanese REFLECT trial, intermediate-stage (i.e., BCLC stage B) patients showed a better ORR with lenvatinib than the BCLC C patients (61.3 vs. $38.0 \%$ ] [30]; our study also showed a better ORR and PFS in patients with BCLC stage B than in those with BCLC stage C (ORR 36.4 vs. 11.1\%; PFS 7.8 vs. 4.3 months). In addition, a previous retrospective analysis suggested that lenvatinib is effective for patients with intermediatestage HCC as an initial therapy [31]. These results may indicate the potential role of lenvatinib for the management of intermediate-stage HCC patients, in whom TACE is generally considered as an initial therapy $[12,32,13-15]$. In future studies, the clinical relevance of lenvatinib should be further investigated for intermediate-stage HCC.

Lenvatinib showed favorable safety profiles in the real-world setting, consistent with the results of the REFLECT trial and previous Japanese retrospective studies. There were no new safety-related events identified in this study. Most AEs were of grade 1 or 2 and were manageable with appropriate supportive care. AST elevation and fatigue, the most frequent AEs, occurred in 52.2 and $39.1 \%$ of patients, respectively, and hyperbilirubinemia $(8.7 \%)$, AST elevation $(6.5 \%)$ and diarrhea $(5.4 \%)$ were the most frequent grade 3-4 AEs. The incidences of hand-foot syndrome, hypothyroidism, and hypertension in our study were lower than in the REFLECT trial. This is mainly due to the lower starting dose in $23.9 \%$ of patients and a potential underestimation considering the nature of our retrospective study design.

Our study had several limitations, the most prominent of which is the retrospective design since this is subject to unintentional biases. Although our study was based on multiple tertiary referral institutions, the number of analyzed patients was relatively small. However, our data are clinically meaningful, as our results offer the first real-world analysis outside of Japan. Our analysis also included patients who were excluded in the pivotal REFLECT trial and those who had received prior systemic therapy including ICIs.

In conclusion, lenvatinib showed consistent real-life efficacy and safety outcomes for Child-Pugh A patients compared to those from the REFLECT trial. Considering the modest efficacy of lenvatinib in Child-Pugh B patients, its use in this group should be cautious. 


\begin{tabular}{l|l|}
\hline Liver Cancer 2020;9:613-624 \\
\hline DOI: 10.1159/000508901 & $\begin{array}{l}\text { @ 2020 The Author(s). Published by S. Karger AG, Basel } \\
\text { www.karger.com/lic }\end{array}$ \\
\hline
\end{tabular}

Cheon et al.: Lenvatinib in Korean Patients with Advanced HCC

\section{Statements of Ethics}

This study was approved by the Institutional Review Board of each participating center (Asan Medical Center, 2019-0977; Ulsan University Hospital, 2019-08-027; CHA Bundang Medical Center, 2019-09-040) and was performed in accordance with the ethics standards of the institutional research committee and the recent Declaration of Helsinki. The need for informed consent in this study was waived, as Korean regulations do not require consent for retrospective analyses.

\section{Conflict of Interest Statement}

J.C. received advisory fees from Eisai and MSD. C.Y. received grants from Bayer and ONO, and consultancy and advisory fees from Bayer, Eisai, Ipsen, and MSD. H.J.C. received consultancy and advisory fees from Bayer, Eisai, ONO, and MSD. The other authors have no potential conflicts of interest related to this article or the research described therein.

\section{Funding Sources}

There was no funding.

\section{Author Contributions}

Study concept and design and manuscript preparation: J.C., H.J.C., C.Y., and B.-Y.R. Data acquisition, analysis, and interpretation, and editing and approval of the manuscript: all authors.

\section{References}

1 Llovet JM, Montal R, Sia D, Finn RS. Molecular therapies and precision medicine for hepatocellular carcinoma. Nat Rev Clin Oncol. 2018 Oct;15(10):599-616.

2 Jung KW, Won YJ, Kong HJ, Lee ES. Cancer Statistics in Korea: Incidence, Mortality, Survival, and Prevalence in 2016. Cancer Res Treat. 2019 Apr;51(2):417-30.

3 Llovet JM, Ricci S, Mazzaferro V, Hilgard P, Gane E, Blanc JF, et al.; SHARP Investigators Study Group. Sorafenib in advanced hepatocellular carcinoma. N Engl J Med. 2008 Jul;359(4):378-90.

4 Cheng AL, Kang YK, Chen Z, Tsao CJ, Qin S, Kim JS, et al. Efficacy and safety of sorafenib in patients in the AsiaPacific region with advanced hepatocellular carcinoma: a phase III randomised, double-blind, placebocontrolled trial. Lancet Oncol. 2009 Jan;10(1):25-34.

5 Cheng AL, Kang YK, Lin DY, Park JW, Kudo M, Qin S, et al. Sunitinib versus sorafenib in advanced hepatocellular cancer: results of a randomized phase III trial. J Clin Oncol. 2013 Nov;31(32):4067-75.

6 Johnson PJ, Qin S, Park JW, Poon RT, Raoul JL, Philip PA, et al. Brivanib versus sorafenib as first-line therapy in patients with unresectable, advanced hepatocellular carcinoma: results from the randomized phase III BRISK-FL study. J Clin Oncol. 2013 Oct;31(28):3517-24.

7 Cainap C, Qin S, Huang WT, Chung IJ, Pan H, Cheng Y, et al. Linifanib versus Sorafenib in patients with advanced hepatocellular carcinoma: results of a randomized phase III trial. J Clin Oncol. 2015 Jan;33(2):172-9.

8 Matsui J, Funahashi Y, Uenaka T, Watanabe T, Tsuruoka A, Asada M. Multi-kinase inhibitor E7080 suppresses lymph node and lung metastases of human mammary breast tumor MDA-MB-231 via inhibition of vascular endothelial growth factor-receptor (VEGF-R) 2 and VEGF-R3 kinase. Clin Cancer Res. 2008 Sep;14(17):545965.

9 Matsuki M, Hoshi T, Yamamoto Y, Ikemori-Kawada M, Minoshima Y, Funahashi Y, et al. Lenvatinib inhibits angiogenesis and tumor fibroblast growth factor signaling pathways in human hepatocellular carcinoma models. Cancer Med. 2018 Jun;7(6):2641-53.

10 Ikeda K, Kudo M, Kawazoe S, Osaki Y, Ikeda M, Okusaka T, et al. Phase 2 study of lenvatinib in patients with advanced hepatocellular carcinoma. J Gastroenterol. 2017 Apr;52(4):512-9.

11 Kudo M, Finn RS, Qin S, Han KH, Ikeda K, Piscaglia F, et al. Lenvatinib versus sorafenib in first-line treatment of patients with unresectable hepatocellular carcinoma: a randomised phase 3 non-inferiority trial. Lancet. 2018 Mar;391(10126):1163-73.

12 European Association for the Study of the Liver. EASL Practical Guidelines: Management of hepatocellular carcinoma. J Hepatol. 2018 Jul;69(1):182-236. 
13 National Comprehensive Cancer Network. [Internet] NCCN Clinical Practice Guidelines in Oncology: Hepatobiliary Cancers. 2019. Available from: https://www.nccn.org/professionals/physician_gls/default.aspx

14 Vogel A, Cervantes A, Chau I, Daniele B, Llovet JM, Meyer T, et al. Correction to: "Hepatocellular carcinoma: ESMO Clinical Practice Guidelines for diagnosis, treatment and follow-up”. Ann Oncol. 2019 May;30(5):871-3.

15 Chen LT, Martinelli E, Cheng AL, Pentheroudakis G, Qin S, Bhattacharyya GS, et al. Pan-Asian adapted ESMO Clinical Practice Guidelines for the management of patients with intermediate and advanced/relapsed hepatocellular carcinoma: a TOS-ESMO initiative endorsed by CSCO, ISMPO, JSMO, KSMO, MOS and SSO. Ann Oncol. 2020 Mar;31(3):334-51.

16 Llovet JM, Brú C, Bruix J. Prognosis of hepatocellular carcinoma: the BCLC staging classification. Semin Liver Dis. 1999;19(3):329-38.

17 Eisenhauer EA, Therasse P, Bogaerts J, Schwartz LH, Sargent D, Ford R, et al. New Response Evaluation Criteria in Solid Tumours: Revised RECIST Guideline (version 1.1). Eur J Cancer. 2009 Jan;45(2):228-47.

18 Kudo M, Finn RS, Qin S, Han K-H, Ikeda K, Cheng A-L, et al. Analysis of survival and objective response (OR) in patients with hepatocellular carcinoma in a phase III study of lenvatinib (REFLECT). J Clin Oncol. 2019;37(4_ suppl):186.

19 Hiraoka A, Kumada T, Atsukawa M, Hirooka M, Tsuji K, Ishikawa T, et al.; Real-life Practice Experts for HCC (RELPEC) Study Group, HCC 48 Group (hepatocellular carcinoma experts from 48 clinics in Japan). Prognostic factor of lenvatinib for unresectable hepatocellular carcinoma in real-world conditions-Multicenter analysis. Cancer Med. 2019 Jul;8(8):3719-28.

20 Terashima T, Yamashita T, Takata N, Toyama T, Shimakami T, Takatori H, et al. Comparative analysis of liver functional reserve during lenvatinib and sorafenib for advanced hepatocellular carcinoma. Hepatol Res. 2020 Jul;50(7):871-84.

21 Hiraoka A, Kumada T, Kariyama K, Takaguchi K, Atsukawa M, Itobayashi E, et al.; Real-Life Practice Experts for HCC (RELPEC) Study Group, HCC 48 Group. Clinical features of lenvatinib for unresectable hepatocellular carcinoma in real-world conditions: multicenter analysis. Cancer Med. 2019 Jan;8(1):137-46.

22 Hiraoka A, Kumada T, Kariyama K, Takaguchi K, Itobayashi E, Shimada N, et al. Therapeutic potential of lenvatinib for unresectable hepatocellular carcinoma in clinical practice: Multicenter analysis. Hepatol Res. 2019 Jan;49(1):111-17.

23 Obi S, Sato T, Sato S, Kanda M, Tokudome Y, Kojima Y, et al. The efficacy and safety of lenvatinib for advanced hepatocellular carcinoma in a real-world setting. Hepatol Int. 2019 Mar;13(2):199-204.

24 Kirino S, Tsuchiya K, Kurosaki M, Kaneko S, Inada K, Yamashita K, et al. Relative dose intensity over the first four weeks of lenvatinib therapy is a factor of favorable response and overall survival in patients with unresectable hepatocellular carcinoma. PLoS One. 2020 Apr;15(4):e0231828.

25 Maruta S, Ogasawara S, Ooka Y, Obu M, Inoue M, Itokawa N, et al. Potential of Lenvatinib for an Expanded Indication from the REFLECT Trial in Patients with Advanced Hepatocellular Carcinoma. Liver Cancer. 2020;1-15.

26 Kim DY, Kim HJ, Han KH, Han SY, Heo J, Woo HY, et al. Real-Life Experience of Sorafenib Treatment for Hepatocellular Carcinoma in Korea: from GIDEON Data. Cancer Res Treat. 2016 Oct;48(4):1243-52.

27 Hiraoka A, Kumada T, Atsukawa M, Hirooka M, Tsuji K, Ishikawa T, et al. Hiraoka A, Kumada T, Atsukawa M, Hirooka M, Tsuji K, Ishikawa T, et al; Real-Life Practice Experts for HCC (RELPEC) Study Group; HCC 48 Group. Important Clinical Factors in Sequential Therapy Including Lenvatinib against Unresectable Hepatocellular Carcinoma. Oncology. 2019;97(5):277-85.

28 Cheng A-L, Qin S, Ikeda M, Galle P, Ducreux M, Zhu A, et al. LBA3IMbrave150: Efficacy and safety results from a ph III study evaluating atezolizumab (atezo) + bevacizumab (bev) vs sorafenib (Sor) as first treatment (tx) for patients (pts) with unresectable hepatocellular carcinoma (HCC). Ann Oncol. 2019;30(Supplement_9).

29 Marrero JA, Kudo M, Venook AP, Ye SL, Bronowicki JP, Chen XP, et al. Observational registry of sorafenib use in clinical practice across Child-Pugh subgroups: the GIDEON study. J Hepatol. 2016 Dec;65(6):1140-7.

30 Yamashita T, Kudo M, Ikeda K, Izumi N, Tateishi R, Ikeda M, et al. REFLECT-a phase 3 trial comparing efficacy and safety of lenvatinib to sorafenib for the treatment of unresectable hepatocellular carcinoma: an analysis of Japanese subset. J Gastroenterol. 2020 Jan;55(1):113-22.

31 Kudo M, Ueshima K, Chan S, Minami T, Chishina H, Aoki T, et al. Lenvatinib as an Initial Treatment in Patients with Intermediate-Stage Hepatocellular Carcinoma Beyond Up-To-Seven Criteria and Child-Pugh A Liver Function: A Proof-Of-Concept Study. Cancers (Basel). 2019 Jul;11(8):E1084.

32 Marrero JA, Kulik LM, Sirlin CB, Zhu AX, Finn RS, Abecassis MM, et al. Diagnosis, Staging, and Management of Hepatocellular Carcinoma: 2018 Practice Guidance by the American Association for the Study of Liver Diseases. Hepatology. 2018 Aug;68(2):723-50. 